\title{
Magnetic Properties of Fe@Pt Nanoparticles with Core/Shell Morphology
}

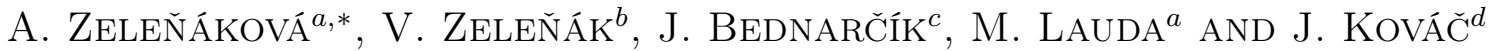 \\ ${ }^{a}$ Department of Solid State Physics, P.J. Šafárik University, Park Angelinum 9, 04154 Košice, Slovakia \\ ${ }^{b}$ Department of Inorganic Chemistry, P.J. Šafárik University, Moyzesova 11, 04001 Košice, Slovakia \\ ${ }^{c}$ DESY-Hasylab, Notkestr. 85, Hamburg, Germany \\ ${ }^{d}$ Institute of Experimental Physics, SAS, Watsonova 47, 04001 Košice, Slovakia
}

\begin{abstract}
We have investigated the magnetic properties of iron nanoparticles coated by platinum shell prepared by chemical procedure. Magnetic measurements using SQUID magnetometer confirm the behaviour typical for a superparamagnetic system. From the $M(H)$ loop at $300 \mathrm{~K}$ the magnetic moment of each particle $m_{\mathrm{P}} \approx 250 \mu_{\mathrm{B}}$ was calculated. The magnetic relaxation study by the in-phase $\chi^{\prime}$ and out-of-phase $\chi^{\prime \prime}$ ac susceptibilities in frequency range $0.1-1000 \mathrm{~Hz}$ suggests the existence of two magnetic layers with different magnetic spin's dynamics and strong dipolar interactions between nanoparticles. The observed magnetic behaviour is caused by the blocking process of interacting superparamagnetic particles with magnetic iron core, diamagnetic platinum shell and spin-glass like behaviour magnetic interlayer between them.
\end{abstract}

PACS numbers: 75.75.Fk, 75.50.Vv, 75.40.Gb

\section{Introduction}

Magnetic nanoparticles exhibit unique properties in comparison with their corresponding bulk analogues, originating from the size and surface effects of the nanostructured materials. At present, the core-shell magnetic nanoparticles are used in a variety of applications e.g. in the fields of medicine (drug delivery systems, in vivo imaging) or data storage (magnetic recording media). The chemically synthesized Fe@Pt nanoparticles are of special interest for future applications in ultrahigh density magnetic storage systems [1-3]. In the presented work we deal with the magnetic properties of Fe@Pt core-shell nanoparticles prepared by chemical procedure.

\section{Experimental}

The particles were synthesized by a reverse micelle method based on the process of the self-assembly of surfactant molecules (CTAB) in octane/water solution. The method allowed us to control the shape of the particles as well as their size by tuning the molar ratio of octane:water. The structural characterization of the nanoparticles was made by powder X-ray diffraction and high resolution transmission electron microscopy (HRTEM) measurements. The X-ray scattering was measured at BW5 Hasylab beamline (DESY Hamburg), at the wavelength

* corresponding author; e-mail: adriana.zelenakova@upjs.sk $\lambda=0.123984 \AA$. Magnetic measurements were performed on a commercial SQUID-based magnetometer (Quantum Design MPMS 5XL) over a wide range of temperatures $(2-300 \mathrm{~K})$ and applied dc fields (up to $50 \mathrm{kOe}$ ). The same instrument was employed for ac susceptibility measurements carried out at frequencies $0.1-1000 \mathrm{~Hz}$ in the temperature region 2-300 K. The samples were encapsulated into a plastic sample holder. The diamagnetic contribution of plastic capsule and plastic sample holder is insignificant in comparison with high magnetic moment of the sample and no correction is necessary.

\section{Results and discussion}

To prepare the nanoparticles with the core@shell structure the several-step chemical process was used. First, Fe nanoparticles were prepared by reverse micelle method and consequently these particles were coated with a layer of Pt. The synthesis was carried out in an Ar atmosphere to prevent oxidation of $\mathrm{Fe}$ particles during preparation. This procedure was reported as useful for preparation of nanoparticles with magnetic core and non-magnetic shell [4].

The temperature and the field dependence of magnetization recorded in temperature range from $2 \mathrm{~K}$ to $300 \mathrm{~K}$ and in external dc magnetic field confirms the superparamagnetic behaviour. The magnetization measured in both protocols zero-fields cooled (ZFC) and field cooled (FC) shows the high irreversibility of ZFC/FC curves. The maximum on ZFC curves is obviously connected with the temperature $T_{\mathrm{B}}$, representing the process 
of blocking/freezing of particle's magnetic moments in external dc field. In our nanoparticles system the unconventional behaviour was observed because of existence of two maxima in ZFC magnetization curve.

The first low temperature maximum at the blocking temperature $T_{\mathrm{B} 1} \approx 5 \mathrm{~K}$ and second maximum $T_{\mathrm{B} 2} \approx$ $25 \mathrm{~K}$ were estimated, see Fig. 1. This behaviour indicates the existence of two magnetic layers with different magnetic spin's dynamics [5].

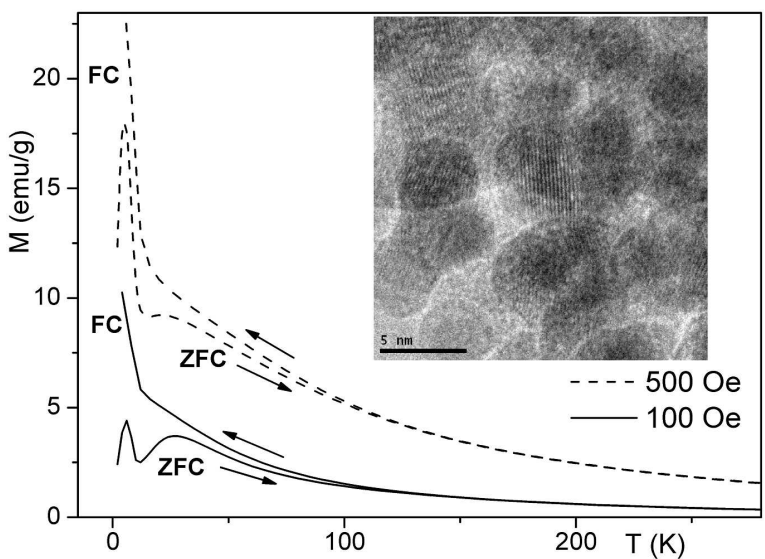

Fig. 1. Temperature dependence of magnetization measured in ZFC and FC protocols in low dc field of 100 Oe (solid line) and 500 Oe (dashed line). Inset: the HRTEM picture of Fe@Pt nanoparticles.

The analysis of field dependences of magnetization at different temperatures confirms that at higher temperatures the moments of particles freely fluctuate in external magnetic field leading to superparamagnetism and below the temperature of $25 \mathrm{~K}$ the magnetic moment of each particle is blocked in the external field direction and leads to the ferromagnetic hysteresis, see Fig. 2. The room temperature magnetization curve was analyzed using the Langevin formalism. The best fit of experimental data to the distribution of the Langevin functions yields the value of particle magnetic moment of $m_{p}=250 \mu_{\mathrm{B}}$. This value related with particle's diameter and calculated value was $d \approx 5 \pm 1 \mathrm{~nm}$.

For the investigation of the dynamic properties and for the estimation of the measure of interparticle interactions we have studied the magnetic properties of nanoparticles in ac magnetic field. The magnetic susceptibility was measured as $\chi(f, T)=\chi^{\prime}(f, T)+\mathrm{i} \chi^{\prime \prime}(f, T)[6]$, where $\chi^{\prime}$ represents in-phase ac susceptibility (real part) and $\chi^{\prime \prime}$ out-of-phase (imaginary part) susceptibility. Data of ac susceptibility measured at frequencies in the range 0.1-1000 Hz exhibit the behaviour typical for blocking/ freezing processes.

Figure 3 demonstrates the presence of a maximum in in-phase ac susceptibility $\chi^{\prime}(T)$ (real part) at the temperature $T_{\mathrm{B}}$, which shifts towards higher temperatures with increasing frequency (see arrow) and the out-of-phase ac susceptibility $\chi^{\prime \prime}(T)$ (imaginary part) ex-

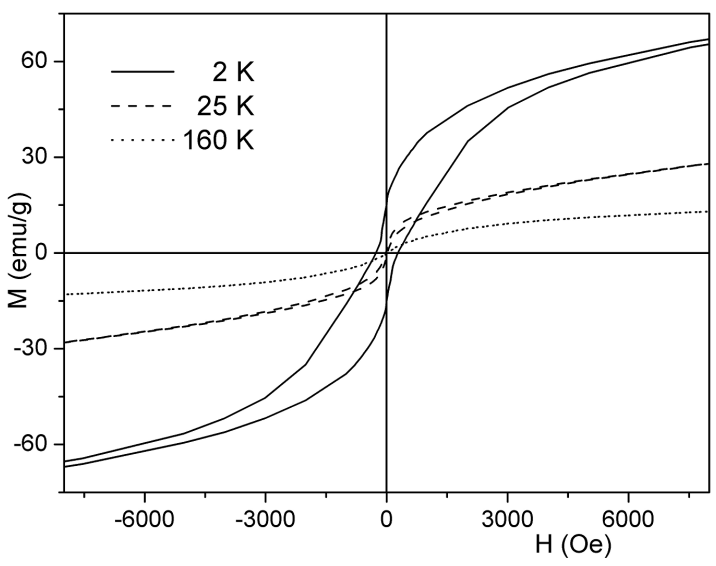

Fig. 2. Field dependence of magnetization measured at different temperatures $2 \mathrm{~K}$ (solid line), $25 \mathrm{~K}$ (dashed line) and $160 \mathrm{~K}$ (dotted line).

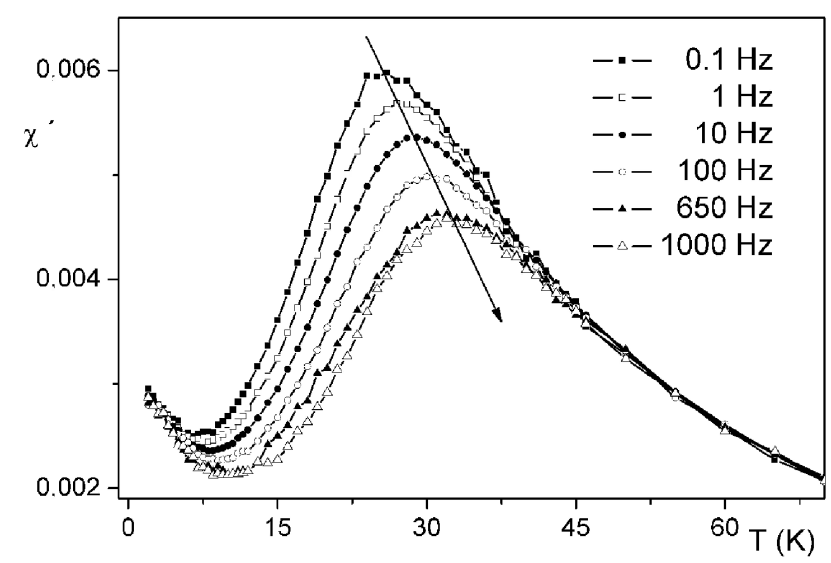

Fig. 3. Temperature dependence of in-phase $\chi^{\prime}(T)$ ac susceptibility (real part) at different frequencies of ac field in the temperature region up to $80 \mathrm{~K}$. The data were taken at $0.1,1,10,100,650$ and $1000 \mathrm{~Hz}$ as indicated in the figure.

hibits a sudden onset near $T_{\mathrm{B}}$. The quantitative analysis of ac susceptibility data due to the Arrhenius law and the Vogel-Fulcher laws confirms the existence of strong inter-particle interactions between nanoparticles. The relaxation time was calculated using relation $\tau=$ $\tau_{0} \exp \frac{E_{A}}{k_{\mathrm{B}}\left(T_{\mathrm{B}}-T_{0}\right)}$, where $E_{A}$ is the energy barrier to magnetization reversal $\left(E_{A} \sim K_{\text {eff }} V\right), T_{\mathrm{B}}$ is the blocking temperature, $\tau_{0}$ is the attempt frequency and parameter $T_{0}$ is an effective temperature that arises from the inter-particle interactions and represents the temperature for which the relaxation time diverges. From the magnetic relaxation studies the values of $\tau_{0} \approx 2 \times 10^{-15} \mathrm{~s}$, $T_{0}=10 \pm 1 \mathrm{~K}, E_{A} / k_{\mathrm{B}}=557$ and effective anisotropy constant $K_{\text {eff }}=2.6 \times 10^{6} \mathrm{~J} / \mathrm{m}^{3}$ were estimated. It was reported that the strong inter-particle interactions were present between superparamagnetic nanoparticles with disordered surface layer [5] due to the strong surface ef- 
fects (caused by surface shell with different spin orientation to the bulk). Our results based on dc and ac magnetic analysis predict the existence of additional magnetic interlayer between magnetic core and diamagnetic shell with different spins orientation to the magnetic core. The spins of this magnetic interlayer can thermally fluctuate and freeze in a disordered spin-glass-like behaviour which is represented by behaviour of the first maximum in ZFC curves. Since the size of particles is small the influence of such different magnetic layers is significant.

\section{Conclusion}

We have synthesized the superparamagnetic spherical Fe@Pt nanoparticles with the core/shell structure. The magnetization measured in both protocols ZFC and FC shows the high irreversibility of ZFC/FC curves. The unconventional magnetic behaviour was observed because of existence of two maxima in $\mathrm{ZFC}$ magnetization curve $\left(T_{\mathrm{B} 1} \approx 5 \mathrm{~K}\right.$ and $\left.T_{\mathrm{B} 2} \approx 25 \mathrm{~K}\right)$, indicating the existence of two magnetic layers with different magnetic spin's dynamics. The inter-particle interactions were investigated using dynamic properties of the system (ac susceptibility). The analysis of ac magnetization study shows strong dipolar interactions between the nanoparticles with the value of characteristic relaxation time $\tau_{0} \approx 2 \times 10^{-15} \mathrm{~s}$, the measure of effective energy barrier $T_{0}=10 \pm 1 \mathrm{~K}$ and the effective anisotropy constant $K_{\text {eff }}=2.6 \times 10^{6} \mathrm{~J} / \mathrm{m}^{3}$.

\section{Acknowledgments}

This work was supported by the projects of Ministry of Education of the Slovak Republic VEGA (No. 1/0311/10, No. $1 / 0138 / 10$, No. $1 / 0119 / 08$ ) and by the ERDF EU (Operational Program "Research and Development" financed through European Regional Development Fund) grants, under the contracts No. ITMS 26220120005 and No. ITMS 26220120019. The authors would like to thank DESY/HASYLAB project under No. I-20100131 EC.

\section{References}

[1] J.P. Wang, Proc. IEEE 96, 1847 (2008).

[2] J.A. Christodoulides, Y. Zhang, G.C. Hadjipanayis, C. Fountzoulas, IEEE Trans. Magn. 36, 2333 (2000).

[3] K. Shinoda, K. Sato, B. Jeyadevan, K. Tohji, IEEE Trans. Magn. 42, 3051 (2006).

[4] K. Sibnath, R.R. Vijayaraghavan, J. Nanosci. Nanotechnol. 10, 5527 (2010).

[5] M. Delalande, P.R. Marcoux, P. Reiss, Y. Samson, J. Mater. Chem. 17, 1579 (2007).

[6] A. Zelenakova, J. Kovac, V. Zelenak, J. Appl. Phys. 108, $034323(2010)$ 